\section{Contextos de vulnerabilidade para o HIV entre mulheres brasileiras}

\author{
Contexts of HIV vulnerability among \\ Brazilian women
}

\author{
${ }_{1}$ Centro de Referência e \\ Treinamento DST/AIDS, \\ Secretaria de Estado da \\ Saúde, São Paulo, Brasil. \\ 2 Núcleo de Estudos de \\ População, Universidade \\ Estadual de Campinas, \\ Campinas, Brasil. \\ 3 Escola Nacional de Saúde \\ Pública Sergio Arouca, \\ Fundação Oswaldo Cruz, \\ Rio de Janeiro, Brasil. \\ 4 Programa de Pós-Graduação \\ em Saúde Coletiva, \\ Universidade Federal de \\ São Paulo, \\ São Paulo, Brasil. \\ 5 Universidade de Franca, \\ Franca, Brasil. \\ Correspondência \\ N.J. S. Santos \\ Centro de Referência e \\ Treinamento DST/AIDS, \\ Secretaria de Estado da \\ Sáude. \\ Rua Santa Cruz 81, \\ São Paulo, SP \\ 04121-000, Brasil. \\ naila@crt.saude.sp.gov.br
}

\begin{abstract}
This article aims to identify contexts of vulnerability related to HIV among Brazilian women. From November 2003 to December 2004, a crosssectional study was conducted in 13 municipalities in the five Brazilian regions. The study included 1,777 women with a positive HIV diagnosis and 2,045 women attending public health care services. There were no significant differences between the two groups concerning number of sexual partners. However, HIV-positive women had a history of earlier sexual initiation and lower frequency of condom use. Higher proportions of HIV-positive women had used drugs, had a history of previous STDs, and had been victims of sexual violence some time in their life. The findings suggest the importance of considering strategies for HIV prevention focused on women's empowerment as a whole, and not focused only on their individual behaviors.
\end{abstract}

Vulnerability; HIV; Acquired Immunodeficiency Syndrome; Women

\author{
Naila J. S. Santos 1 \\ Regina Maria Barbosa 1,2 \\ Adriana A. Pinho 3 \\ Wilza V. Villela 4,5 \\ Tirza Aidar 2 \\ Elvira M. V. Filipe 1
}

\section{Introdução}

Desde seu início, o cenário da epidemia de HIV/ AIDS vem se modificando no Brasil e no mundo, o que se reflete em alterações do perfil epidemiológico das pessoas vivendo com HIV/AIDS. Nos primeiros anos da epidemia os homens representavam a imensa maioria dos casos, e os coeficientes de incidência no sexo masculino eram, expressivamente, maiores do que no sexo feminino. Entretanto, embora mais indivíduos do sexo masculino no total de casos de AIDS sejam notificados no Brasil, a velocidade de crescimento da epidemia é, como em outros países, maior entre as mulheres do que entre os homens. $\mathrm{O}$ fato pode ser atestado pela queda progressiva da relação masculino/feminino de casos e a redução mais expressiva da mortalidade por AIDS entre os homens do que entre as mulheres 1 .

Quando se analisa a evolução da epidemia no sexo feminino no país, observam-se três fases distintas em termos de risco para a infecção pelo HIV: a primeira fase, até 1986, quando a transmissão pela via sexual era a mais importante, sendo, naquele momento, as parcerias com homens que fazem sexo com homens (HSH) e homens transfundidos as mais freqüentes. Nesse período era também relevante a transmissão pela transfusão sangüínea 2. A segunda fase, do fim da década de 80 ao início da década de 90, em que o uso de drogas injetáveis aparece como uma importante forma de infecção pelo HIV, particularmente 
na Região Sudeste do país 2; e a terceira fase, do início dos anos 90 até o presente momento ${ }^{1}$, que apresenta nítido predomínio da prática heterossexual como forma de transmissão do HIV para as mulheres.

A investigação sobre a probabilidade de transmissão do HIV nas relações heterossexuais tem indicado que a chance de transmissão homem-mulher é maior do que a transmissão mulher-homem, ainda que não haja consenso sobre a magnitude dessa diferença 3,4. Apesar dos diversos fatores biológicos apontados para explicar a maior chance de infecção pelo HIV entre mulheres 5 , não se pode subestimar a forte associação das relações de gênero nas sociedades com a maior vulnerabilidade das mulheres à infecção pelo HIV 6,7.

O perfil dos casos notificados de AIDS entre mulheres no país remete a alguns fatores que contribuem para a sua vulnerabilidade: o fato de as mulheres com HIV terem menor escolaridade que os homens em igual situação, entendida esta menor escolaridade como proxy de uma condição sócio-econômica menos privilegiada e, ainda, uma maior proporção de mulheres que apresentam como categoria de exposição para o HIV o fato de seus parceiros terem múltiplas parceiras sexuais. Considere-se que no país o nível médio de escolaridade das mulheres é discretamente mais alto que o dos homens (Instituto Brasileiro de Geografia e Estatística. Censo Demográfico, 2000. http://www.ibge.gov.br) e que entre os homens heterossexuais existe uma maior proporção de multiplicidade de parcerias. Ou seja, o perfil das mulheres com HIV reitera que a prática de multiplicidade de parceiros é mais freqüente e socialmente aceitável nos homens do que nas mulheres, e mesmo quando estas não concordam com o comportamento dos seus parceiros, nem sempre dispõem de meios materiais e simbólicos para sair da relação ou mudar os seus termos 8 .

Diversos aspectos ligados às relações sociais de gênero determinam um baixo poder de negociação sexual das mulheres, tornando-as mais propensas a terem relações sexuais desprotegidas aumentando, conseqüentemente, as suas chances de exposição ao HIV. A submissão das mulheres aos homens no que diz respeito ao exercício da sexualidade e a sua responsabilização pelas questões reprodutivas - contracepção e concepção - dificulta o diálogo com seus parceiros e aumenta a vulnerabilidade das mulheres. Diante disso, não é possível pensar em ações de prevenção sem considerar as relações de gênero enquanto relações de poder.

Segundo Barbosa 9 (p. 281), “a vulnerabilidade feminina ao HIV necessariamente remete às formas como homens e mulheres se relacionam em nossa sociedade, a dinâmica de poder que perpassa tais relações e o imaginário coletivo em relação aos papéis de gênero - que, certamente, constituem importantes variáveis na conformação do atual perfil da epidemia".

Os modelos propostos de prevenção derivados do início da epidemia, em cujo perfil predominavam os homens gays, precisam ser revistos e adaptados. Os avanços da terapia anti-retroviral e as tendências atuais da epidemia devem levar a reflexões sobre novas formas de abordagem do HIV/AIDS, buscando a implementação e manutenção de políticas públicas de prevenção e assistência cada vez mais eficientes, com o desafio e a obrigação moral e ética de torná-las acessíveis a toda a população.

Este trabalho tem por objetivo traçar um perfil das mulheres vivendo com HIV/AIDS no Brasil, buscando identificar os fatores que as particularizam, visando subsidiar propostas de intervenções mais eficientes em termos da prevenção da infecção e do cuidado à saúde sexual e reprodutiva das mulheres atingidas pela epidemia de AIDS.

\section{Metodologia}

Entre novembro de 2003 e dezembro de 2004, um estudo de corte transversal foi conduzido com 3.822 mulheres acima de 17 anos, em 13 municípios brasileiros, divididas em dois grupos: 1.777 mulheres com diagnóstico positivo para HIV e 2.045 mulheres usuárias de serviços públicos de atenção à saúde da mulher sem diagnóstico conhecido de soropositividade para o HIV. Para ambos os grupos, utilizou-se uma amostra de conveniência de serviços localizados no Distrito Federal e em capitais ou municípios de grande porte das cinco regiões brasileiras: Norte, Nordeste, Centro-Oeste, Sudeste e Sul. Os municípios escolhidos foram: Belém (Pará), Campina Grande (Paraíba), Recife (Pernambuco), Vitória da Conquista (Bahia), São Paulo, Ribeirão Preto (São Paulo), Rio de Janeiro, Belo Horizonte (Minas Gerais), Goiânia (Goiás), Campo Grande (Mato Grosso do Sul), Curitiba (Paraná) e Pelotas (Rio Grande do Sul).

O grupo de mulheres vivendo com HIV/AIDS foi selecionado em serviços de atendimento especializado em DST/AIDS. O cálculo do tamanho da amostra foi feito utilizando-se uma estimativa da soroprevalência do HIV na população por macrorregião, para um intervalo de $95 \%$ de confiança (IC95\%) e erro bilateral de 5\%. Na Região Norte, utilizou-se um erro bilateral de 7,5\% para o grupo de mulheres HIV positivo porque não 
seria possível obter uma amostra maior na região, em razão do menor número de pessoas atendidas nos serviços de atendimento especializado.

A seleção do grupo comparativo foi realizada nos mesmos municípios acima listados, em unidades básicas de saúde e em serviços de atenção à saúde da mulher que possuíssem mais de $50 \%$ dos seus atendimentos voltados às consultas de pré-natal e/ou planejamento familiar. A seleção dos serviços em cada município também foi por conveniência mediante convite para constituírem sítios de pesquisa. Além de pertencerem a redes administrativas diferenciadas, quer no plano da rede pública estadual, quer no plano das redes municipais, também contemplaram diferentes situações de entrada e constituição da clientela quanto à demanda (espontânea, estimulada ou referida) e modelos de assistência.

As mulheres vivendo com HIV/AIDS foram consideradas elegíveis para participação no estudo se tivessem 18 anos ou mais e soubessem ler e escrever, uma vez que o instrumento de pesquisa era auto-aplicável. O profissional de saúde responsável pelo convite às mulheres em cada unidade foi instruído a convidar apenas as mulheres com exames sabidamente soropositivos, matriculadas no respectivo serviço. As mulheres usuárias dos serviços de saúde da mulher foram consideradas elegíveis se tivessem 18 anos ou mais e soubessem ler e escrever, independentemente do conhecimento do seu estado sorológico quanto à infecção pelo HIV. Embora o conhecimento do estado sorológico em relação ao HIV não fosse critério de elegibilidade, no instrumento de coleta de dados era perguntado sobre a realização do teste anti-HIV alguma vez na vida, e se sabiam o resultado. Apenas uma mulher respondeu que seu resultado era positivo e foi excluída deste grupo. Como não houve confirmação do estado sorológico das mulheres que nunca fizeram o teste e daquelas que fizeram e responderam que eram soronegativas ou que não sabiam sua sorologia, esse grupo será referido como mulheres não vivendo com HIV/AIDS.

O convite para participar do estudo ocorria no momento da consulta, agendada ou espontânea, das usuárias dos serviços selecionados e era feito pelo profissional do serviço treinado para tal abordagem. Após esclarecimento dos objetivos da pesquisa, para as mulheres que concordassem em participar eram entregues os questionários, anônimos e auto-aplicáveis. Após seu preenchimento, que podia ser realizado enquanto aguardava a consulta, a mulher era orientada a depositá-lo em uma urna lacrada com numeração específica para cada serviço. O profissional responsável pela pesquisa em cada serviço recolhia os questionários da urna e os enviava para a coordenação central da pesquisa para a codificação e dupla digitação dos dados.

Foram utilizados dois questionários semiestruturados, um para cada grupo estudado, contendo os seguintes blocos de questões: (a) sócio-demográfico - idade, município e região de residência (macrorregião), cor da pele, escolaridade, trabalho, fonte de renda, e estado conjugal; (b) comportamento e saúde sexual - idade na primeira relação sexual, número de parceiros sexuais na vida e nos últimos seis meses, tipo de parceria (fixa e ocasional) e histórico de doença sexualmente transmissível (DST); (c) comportamento contraceptivo e reprodutivo - uso e tipo de métodos contraceptivos, número de filhos e intenção reprodutiva (desejo e intenção de ter filhos ou mais filhos); (d) situações de risco para DST/AIDS - uso de preservativo em relações sexuais, uso de drogas pela entrevistada e pelo parceiro sexual, e história de prática de sexo em troca de dinheiro e/ou drogas; (e) acesso aos serviços de saúde - consulta ginecológica nos últimos 12 meses, realização do exame de Papanicolaou nos últimos 12 meses, qualidade do atendimento recebido e dificuldades funcionais do serviço de saúde, como longo período de espera para ser atendida ou marcar uma consulta, entre outros. Para este artigo, apenas variáveis dos blocos (a) ao (d) foram utilizadas.

Entre as mulheres vivendo com HIV/AIDS, além das questões acima referidas, investigou-se a história da infecção pelo HIV e do diagnóstico: idade com que achava que se infectou, idade na época do diagnóstico, tempo (auto-referido) desde o conhecimento da soropositividade, forma auto-percebida de infecção pelo HIV, forma de conhecimento sobre a infecção e uso de terapia anti-retroviral (TARV), além da sorologia do parceiro para aquelas com parceria fixa.

O questionário foi pré-testado quanto à sua extensão e à facilidade de compreensão das questões e do autopreenchimento. Os dados foram duplamente digitados e a análise foi realizada pela utilização do pacote estatístico Stata 9.1 (Stata Corp., College Station, Estados Unidos).

Para a análise dos dados, realizou-se inicialmente uma caracterização da amostra de mulheres não vivendo com HIV/AIDS e mulheres vivendo com HIV/AIDS, comparando as características sócio-demográficas das mulheres no momento da entrevista em cada grupo estudado. Análises descritivas de algumas características relacionadas à história da infecção e do diagnóstico também foram realizadas com a apresentação das medidas de freqüência, de tendência central e de dispersão.

Para análise comparativa do contexto de vulnerabilidade ao HIV entre os dois grupos de 
mulheres, foram consideradas as variáveis sobre o comportamento sexual e reprodutivo de mulheres vivendo com HIV/AIDS referentes à época em que achavam que haviam se infectado. Por mais que esse momento seja de difícil identificação, a opção por esta estratégia metodológica teve como objetivo uma maior aproximação com o contexto no qual ocorreu a infecção, tal como proposto por Bajos et al. 10 ao estudar outro tipo de desfecho relacionado à atividade sexual, o aborto. Segundo a autora, eventos relacionados à vida sexual das mulheres, como é o caso da maioria dos relacionados à infecção pelo HIV, só podem ser analisados tendo como referência os contextos específicos que compõem os diferentes momentos de suas trajetórias sexual, reprodutiva e social.

Além disso, para permitir maior comparabilidade entre os grupos, as proporções foram ajustadas por padronização direta por meio dos cinco estratos etários criados (18-24 anos, 25-29 anos, 30-34 anos, 35-39 anos e 40 anos ou mais). As diferenças entre os grupos foram analisadas pelo teste de associação do qui-quadrado de Pearson (ou teste exato de Fisher) para variáveis categóricas e do teste t de Student ou do teste não paramétrico de Mann-Whitney para as variáveis contínuas.

Este estudo foi aprovado pelo Comitê de Ética em Pesquisa do Centro de Referência e Treinamento DST/AIDS da Secretaria Estadual de Saúde de São Paulo, onde foi sediada a coordenação do mesmo, assim como por alguns comitês de ética locais, conforme a demanda dos municípios.

\section{Resultados}

As características sócio-demográficas do grupo de mulheres vivendo e não vivendo com HIV/ AIDS no momento da entrevista são apresentadas na Tabela 1. Comparativamente às mulheres não vivendo com HIV/AIDS, mulheres vivendo com HIV/AIDS eram mais velhas, com menor escolaridade e uma proporção maior delas não estava em união estável e não trabalhava à época da pesquisa ( $p<0,0001)$. Não houve diferenças estatisticamente significantes em relação à cor da pele entre os grupos; $46 \%$ de mulheres de ambos os grupos se autodeclararam brancas, $34 \%$ pardas e ao redor de $15 \%$ negras. Quanto à região de residência, proporções similares entre mulheres vivendo com HIV/AIDS e mulheres não vivendo com HIV/AIDS foram observadas para aquelas residentes nas regiões Nordeste, Centro-Oeste e Sul, com exceção das regiões Norte e Sudeste, onde se observou uma sub e sobre-representação de mulheres vivendo com HIV/AIDS, respectiva- mente. A sub-representação de mulheres vivendo com HIV/AIDS na Região Norte deveu-se a problemas ocorridos durante a coleta dos dados em um dos serviços de saúde.

Entre as mulheres vivendo com HIV/AIDS as principais formas autopercebidas de infecção foram: o parceiro ter múltiplas parceiras sexuais ou ser bissexual (38\%), elas terem tido relações sexuais desprotegidas (23\%) e o parceiro ser usuário de drogas $(17,3 \%)$ (Tabela 2 ). Apenas $4,2 \%$ das mulheres vivendo com HIV/AIDS referiram que achavam que haviam se infectado por ser ou ter sido profissional do sexo.

$\mathrm{O}$ número mediano de meses desde o diagnóstico da infecção pelo HIV foi de 36 meses, excluindo-se $18 \%$ daquelas que não responderam sobre o tempo desde o diagnóstico; $20,2 \%$ da amostra tiveram conhecimento de seu status sorológico nos últimos 12 meses anteriores à entrevista. A idade mediana na época da infecção foi de 27 anos com uma diferença de dois anos para a idade mediana na época do diagnóstico (29 anos).

Das 1.098 mulheres vivendo com HIV/AIDS que responderam ter parceiro fixo no momento da entrevista, $82,4 \%$ conheciam a sorologia de seus parceiros, e 46,3\% afirmaram que o seu parceiro também era portador do HIV. Entre as mulheres vivendo com HIV/AIDS com parceiro fixo na época da infecção, $70 \%$ relataram que foi este parceiro que as infectou e $22 \%$ não sabiam se haviam sido infectadas pelo parceiro ou não.

Com relação à forma de conhecimento do diagnóstico, as principais situações referidas foram: o marido ou namorado ficou doente $(24,7 \%)$, ela ficou doente com sintomas de HIV/ AIDS (24,6\%), soube pelo teste sorológico realizado no pré-natal $(19,5 \%)$ e teste realizado por vontade própria $(12,7 \%)$.

Quanto ao uso de TARV, a maioria das mulheres vivendo com HIV/AIDS respondeu que fazia uso no momento da entrevista (72,9\%).

Não houve diferenças estatisticamente significantes quanto à ocorrência de parceria fixa ou eventual ao longo da vida entre mulheres não vivendo com HIV/AIDS e mulheres vivendo com HIV/AIDS (Tabela 3). No entanto, a proporção de mulheres vivendo com HIV/AIDS casadas ou que moravam com o parceiro diminuiu significantemente de $66,1 \%$ na época da infecção para $51,7 \%$ na época da entrevista (Tabela 1).

Quanto às características de comportamento sexual, as mulheres vivendo com HIV/AIDS, comparativamente às mulheres não vivendo com HIV/AIDS, foram mais jovens na primeira relação sexual, com $34,8 \%$ de mulheres vivendo com HIV/AIDS referindo ter iniciado atividade sexual antes dos 16 anos contra 23,4\% das mulheres 
Características sócio-demográficas de mulheres não vivendo com HIV/AIDS ( $n=2.045)$ e mulheres vivendo com HIV/AIDS ( $n=1.777$ ) de cinco regiões brasileiras, 2003-2004.

\begin{tabular}{|c|c|c|c|c|}
\hline \multirow[t]{2}{*}{ Características } & \multicolumn{2}{|c|}{$\begin{array}{c}\text { Mulheres não vivendo } \\
\text { com HIV/AIDS }\end{array}$} & \multicolumn{2}{|c|}{$\begin{array}{l}\text { Mulheres vivendo } \\
\text { com HIV/AIDS }\end{array}$} \\
\hline & $n$ * & $\%$ & $n$ * & $\%$ \\
\hline \multicolumn{5}{|l|}{ Região de residência } \\
\hline Norte & 221 & 10,8 & 113 & 6,4 \\
\hline Nordeste & 474 & 23,2 & 371 & 20,9 \\
\hline Centro-Oeste & 481 & 23,5 & 352 & 19,8 \\
\hline Sul & 410 & 20,1 & 379 & 21,3 \\
\hline Sudeste & 459 & 22,4 & 562 & 31,6 \\
\hline \multicolumn{5}{|l|}{ Idade (anos) ** } \\
\hline $18-24$ & 594 & 29,7 & 216 & 12,5 \\
\hline $25-29$ & 383 & 19,1 & 328 & 18,9 \\
\hline $30-34$ & 277 & 13,8 & 367 & 21,2 \\
\hline $35-39$ & 235 & 11,7 & 328 & 18,9 \\
\hline 40 ou mais & 514 & 25,7 & 493 & 28,5 \\
\hline Mediana (intervalo interquartil) & \multicolumn{2}{|c|}{$30(24-40)$} & \multicolumn{2}{|c|}{$34(28-40)$} \\
\hline \multicolumn{5}{|l|}{ Educação ** } \\
\hline Nunca foi à escola & 28 & 1,4 & 57 & 3,3 \\
\hline 1ạ a 4ạ série do Ensino Fundamental & 328 & 16,2 & 492 & 28,1 \\
\hline 5a a 8a série do Ensino Fundamental & 631 & 31,2 & 606 & 34,6 \\
\hline Ensino Médio incompleto/completo & 798 & 39,5 & 465 & 26,5 \\
\hline Ensino Superior incompleto/completo & 236 & 11,7 & 132 & 7,5 \\
\hline \multicolumn{5}{|l|}{ Cor da pele } \\
\hline Branca & 907 & 46,6 & 760 & 45,7 \\
\hline Parda & 639 & 32,9 & 573 & 34,5 \\
\hline Negra & 279 & 14,3 & 244 & 14,7 \\
\hline Amarela & 69 & 3,6 & 47 & 2,8 \\
\hline Indígena & 51 & 2,6 & 38 & 2,3 \\
\hline \multicolumn{5}{|l|}{ Cohabitação com parceiro ** } \\
\hline Não & 513 & 26,2 & 827 & 48,3 \\
\hline Sim & 1443 & 73,8 & 885 & 51,7 \\
\hline \multicolumn{5}{|l|}{ Trabalha atualmente ** } \\
\hline Não & 1029 & 50,6 & 1221 & 69,8 \\
\hline Sim & 1003 & 49,4 & 528 & 30,2 \\
\hline
\end{tabular}

* Números diferem para cada variável por causa da não-resposta;

** Diferenças entre mulheres vivendo com HIV/AIDS e mulheres não vivendo com HIV/AIDS estatisticamente significantes $(p<0,05)$.

não vivendo com HIV/AIDS ( $\mathrm{p}<0,05)$ (Tabela 3 ). Apenas oito mulheres, sete entre mulheres não vivendo com HIV/AIDS e uma mulher vivendo com HIV/AIDS, referiram nunca ter tido relação sexual na vida. Sessenta e seis mulheres vivendo com HIV/AIDS (3,8\%) referiram ter tido relação sexual com homens e mulheres na vida e apenas 17 mulheres não vivendo com HIV/AIDS (0,8\%) referiram o mesmo comportamento. Ao todo, apenas sete mulheres relataram comportamento exclusivamente homossexual na vida.
Não houve diferença significante entre o número mediano de parceiros sexuais referidos na vida pelas mulheres não vivendo com HIV/AIDS e até o momento da infecção pelas mulheres vivendo com HIV/AIDS, excluindo-se $4 \%$ de mulheres não vivendo com HIV/AIDS e 5\% de mulheres vivendo com HIV/AIDS que responderam "muitos parceiros" ou "não sabe" da análise. Contudo, considerando ou não esses casos na categoria quatro ou mais parceiros sexuais na vida, uma proporção um pouco maior de mulheres 
Caracterização da amostra de mulheres vivendo com HIV/AIDS ( $n=1.777)$ segundo alguns aspectos de sua infecção e diagnóstico em cinco regiões brasileiras, 2003-2004.

\begin{tabular}{|c|c|c|}
\hline Características & $n$ * & $\%$ \\
\hline \multicolumn{3}{|c|}{ Tempo desde o diagnóstico de soropositividade (anos) [n = 1.457] } \\
\hline $0-1$ & 295 & 20,2 \\
\hline $2-3$ & 449 & 30,8 \\
\hline $4-6$ & 317 & 21,7 \\
\hline 7 ou mais & 396 & 27,3 \\
\hline Mediana (intervalo interquartil) & $3(2-6)$ & \\
\hline \multicolumn{3}{|l|}{ Idade na época do diagnóstico (anos) [n = 1.432] } \\
\hline 24 ou menos & 411 & 28,7 \\
\hline $25-29$ & 347 & 24,2 \\
\hline $30-34$ & 273 & 19,1 \\
\hline $35-39$ & 194 & 13,5 \\
\hline 40 ou mais & 207 & 14,5 \\
\hline Mediana (intervalo interquartil) & $29(23-36)$ & \\
\hline \multicolumn{3}{|l|}{ Idade na época da infecção (anos) [n = 1.608] } \\
\hline 24 ou menos & 576 & 37,0 \\
\hline $25-29$ & 346 & 22,2 \\
\hline $30-34$ & 256 & 16,5 \\
\hline $35-39$ & 184 & 11,8 \\
\hline 40 ou mais & 194 & 12,5 \\
\hline Mediana (intervalo interquartil) & $27(22-35)$ & \\
\hline \multicolumn{3}{|l|}{ Parceiro fixo atual HIV positivo [ $n=1.098]$ ** } \\
\hline Não & 396 & 36,1 \\
\hline Sim & 509 & 46,3 \\
\hline Não sei & 193 & 17,6 \\
\hline \multicolumn{3}{|l|}{ Parceiro fixo quem Ihe infectou $[n=1.429] * *$} \\
\hline Não & 113 & 8,0 \\
\hline Sim & 1.000 & 70,0 \\
\hline Não sei & 316 & 22,0 \\
\hline \multicolumn{3}{|l|}{ Forma autopercebida de infecção [ $n=1.708]$} \\
\hline Parceiro com múltiplas parceiras ou bissexual & 646 & 38,0 \\
\hline Tem ou teve relações sexuais desprotegidas & 392 & 23,0 \\
\hline Parceiro usuário de drogas & 295 & 17,3 \\
\hline Uso de drogas alguma vez na vida & 88 & 5,2 \\
\hline Múltiplos parceiros na vida & 93 & 5,4 \\
\hline É ou já foi profissional do sexo & 72 & 4,2 \\
\hline Transfusão de sangue & 77 & 4,5 \\
\hline Através de violência sexual & 49 & 2,8 \\
\hline \multicolumn{3}{|l|}{ Forma de conhecimento da soropositividade [n = 1.684] } \\
\hline Meu marido ou namorado ficou doente & 417 & 24,7 \\
\hline Fiquei doente com sintomas de HIV/AIDS & 415 & 24,6 \\
\hline Descobri pelos exames do pré-natal & 328 & 19,5 \\
\hline Fiz o teste por vontade própria & 214 & 12,7 \\
\hline Meu médico pediu para eu fazer o teste & 150 & 8,9 \\
\hline Meu filho ficou doente com sintomas de HIV/AIDS & 59 & 3,5 \\
\hline Fizeram o teste sem me avisar & 25 & 1,5 \\
\hline Outra forma & 76 & 4,5 \\
\hline \multicolumn{3}{|l|}{ Uso de terapia anti-retroviral $[n=1.777]$} \\
\hline Não & 481 & 27,1 \\
\hline Sim & 1.296 & 72,9 \\
\hline
\end{tabular}

* Números diferem para cada variável por causa da não-resposta;

** Excluída desta análise uma mulher que nunca teve relação sexual (1 caso). 
Características sexuais, reprodutivas e comportamentais de mulheres não vivendo com HIV/AIDS ( $n=2.045)$ e mulheres vivendo com HIV/AIDS ( $n=1.777$ ) de cinco regiões brasileiras, 2003-2004.

\begin{tabular}{|c|c|c|c|c|}
\hline \multirow[t]{2}{*}{ Características } & \multicolumn{2}{|c|}{$\begin{array}{c}\text { Mulheres não vivendo } \\
\text { com HIV/AIDS } \\
\text { Atualmente * }\end{array}$} & \multicolumn{2}{|c|}{$\begin{array}{c}\text { Mulheres vivendo } \\
\text { com HIV/AIDS } \\
\text { Na época da infecção * }\end{array}$} \\
\hline & $n * \star$ & $\% * \star * *$ & $\mathrm{n} * *$ & 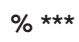 \\
\hline \multicolumn{5}{|c|}{ Idade na primeira relação sexual (anos) \# } \\
\hline Menos de 16 & 477 & 23,4 & 653 & 34,8 \\
\hline $16-17$ & 522 & 26,3 & 431 & 25,4 \\
\hline 18 mais & 939 & 50,3 & 590 & 39,8 \\
\hline Mediana (interval interquartil) & \multicolumn{2}{|c|}{$17(16-19)$} & \multicolumn{2}{|c|}{$16(15-18)$} \\
\hline \multicolumn{5}{|c|}{ Número de parceiros sexuais na vida \# } \\
\hline 1 & 762 & 39,9 & 490 & 32,3 \\
\hline $2-3$ & 606 & 32,3 & 528 & 35,6 \\
\hline 4 ou mais /não sabe & 499 & 27,8 & 504 & 32,1 \\
\hline Mediana (interval interquartil) & \multicolumn{2}{|c|}{$2(1-3)$} & \multicolumn{2}{|c|}{$2(1-4)$} \\
\hline \multicolumn{5}{|l|}{ Coabitação com parceiro \# } \\
\hline Não & 506 & 25,0 & 584 & 33,9 \\
\hline Sim & 1.443 & 75,0 & 1.074 & 66,1 \\
\hline \multicolumn{5}{|l|}{ Tem/Tinha parceiro fixo } \\
\hline Não & 260 & 13,1 & 250 & 14,3 \\
\hline Sim & 1624 & 86,9 & 1436 & 85,7 \\
\hline \multicolumn{5}{|l|}{ Tem/Teve parceiros eventuais } \\
\hline Não & 1.451 & 77,5 & 1.261 & 77,0 \\
\hline Sim & 416 & 22,5 & 392 & 23,0 \\
\hline \multicolumn{5}{|l|}{ Número de filhos vivos \#\# } \\
\hline Nenhum & 431 & 19,8 & 430 & 21,2 \\
\hline 1 & 497 & 25,3 & 446 & 23,9 \\
\hline 2 & 501 & 27,3 & 401 & 24,7 \\
\hline 3 ou mais & 518 & 27,6 & 424 & 30,2 \\
\hline \multicolumn{5}{|l|}{ Uso de preservativo \#,\#\#\# } \\
\hline Nunca & 579 & 32,0 & 1.001 & 62,2 \\
\hline Sempre & 404 & 21,9 & 105 & 5,5 \\
\hline Ás vezes & 852 & 46,1 & 585 & 32,3 \\
\hline \multicolumn{5}{|l|}{ Prática de uso de drogas \#,§ } \\
\hline Não & 1.966 & 96,6 & 1.563 & 94,7 \\
\hline $\operatorname{Sim}$ & 72 & 3,4 & 118 & 5,3 \\
\hline \multicolumn{5}{|c|}{ Prática de sexo em troca de dinheiro } \\
\hline \multicolumn{5}{|l|}{ ou drogas \#, } \\
\hline Não & 2.003 & 98,3 & 1.523 & 92,8 \\
\hline Sim & 35 & 1,7 & 142 & 7,2 \\
\hline
\end{tabular}

(continua)

vivendo com HIV/AIDS, comparativamente às mulheres não vivendo com HIV/AIDS, relatou ter tido quatro ou mais parceiros sexuais até o momento da infecção. Ao excluir as mulheres que relataram sexo em troca de dinheiro e/ou drogas desta análise para ambos os grupos $(\mathrm{n}=177)$, a diferença entre as mulheres vivendo com HIV/ AIDS e as mulheres não vivendo com HIV/AIDS com quatro ou mais parceiros sexuais passou a não ser mais estatisticamente significante. Embora estes dados não constem da Tabela 3, cabe salientar que $15 \%$ das mulheres vivendo com HIV/AIDS com quatro ou mais parceiros sexuais referiram terem feito sexo em troca de dinheiro e/ou drogas, e esta proporção foi significativamente menor para as mulheres não vivendo com HIV/AIDS $(5 \%$; $<0,05)$. 


\begin{tabular}{|c|c|c|c|c|}
\hline \multirow[t]{3}{*}{ Características } & \multirow{2}{*}{\multicolumn{2}{|c|}{$\begin{array}{l}\text { Mulheres não vivendo } \\
\text { com HIV/AIDS } \\
\text { Atualmente * }\end{array}$}} & \multirow{2}{*}{\multicolumn{2}{|c|}{$\begin{array}{c}\text { Mulheres vivendo } \\
\text { com HIV/AIDS } \\
\text { Na época da infecção * }\end{array}$}} \\
\hline & & & & \\
\hline & $\mathrm{n} * *$ & $\%$ *** & $\mathrm{n} * *$ & $\%$ \%** \\
\hline \multicolumn{5}{|c|}{ Ocorrência de DST (auto-referida) \# } \\
\hline Não & 1.572 & 80,0 & 1.191 & 72,7 \\
\hline Sim & 326 & 16,7 & 373 & 21,0 \\
\hline Não lembra & 62 & 3,3 & 110 & 6,3 \\
\hline \multicolumn{5}{|c|}{ Violência sexual na vida \# } \\
\hline Não & 1.718 & 86,8 & 1.303 & 77,9 \\
\hline Sim & 249 & 13,2 & 398 & 22,1 \\
\hline \multicolumn{5}{|c|}{$\begin{array}{l}\text { * Excluídas da análise mulheres que nunca tiveram relação sexual (7 mulheres não vivendo com HIV/AIDS e } 1 \text { mulher vivendo } \\
\text { com HIV/AIDS); }\end{array}$} \\
\hline \multicolumn{5}{|c|}{ ** Números diferem para cada variável por causa da não-resposta; } \\
\hline \multicolumn{5}{|c|}{ *** As proporções foram padronizadas por idade; } \\
\hline \multicolumn{5}{|c|}{ \# Diferenças entre os grupos estatisticamente significantes $(p<0,05)$; } \\
\hline \multirow{2}{*}{\multicolumn{5}{|c|}{$\begin{array}{l}\text { \#\# Para as mulheres vivendo com HIV/AIDS, número de filhos na época da infecção se refere ao número de filhos quando } \\
\text { fizeram o teste de HIV; }\end{array}$}} \\
\hline & & & & \\
\hline \multirow{2}{*}{\multicolumn{5}{|c|}{$\begin{array}{l}\text { \#\#\# Mulheres não vivendo com HIV/AIDS sem atividade sexual nos últimos seis meses prévios à pesquisa foram excluídas da } \\
\text { análise ( } 90 \text { casos); }\end{array}$}} \\
\hline & & & & \\
\hline$\S$ Esta variável se r & da para $n$ & vivend & IDS e pr & ca da infecção \\
\hline
\end{tabular}

A porcentagem de não-resposta para a variável "número de parceiros sexuais na vida" foi, respectivamente, de $14,3 \%(\mathrm{n}=255)$ para mulheres vivendo com HIV/AIDS e 8,7\% $(n=178)$ para mulheres não vivendo com HIV/AIDS $(\mathrm{p}<0,05)$. Não houve diferenças entre os grupos quanto à idade e escolaridade das não respondentes. Além disso, entre mulheres vivendo com HIV/AIDS não respondentes sobre o número de parceiros sexuais até o momento da infecção, investigou-se o relato sobre o número de parceiros sexuais referidos até o momento da entrevista entre aquelas que também responderam tal questão $(\mathrm{n}=161)$. Não houve diferenças estatisticamente significantes quanto ao número de parceiros sexuais até o momento da entrevista entre tais mulheres, fato que poderia sugerir que a não-resposta sobre o número de parceiros sexuais até o momento da infecção poderia ter acontecido devido a um viés de memória em função do tempo decorrido desde a infecção, e não a um comportamento diferenciado.

Não houve diferenças estatisticamente significantes entre mulheres vivendo com HIV/AIDS e mulheres não vivendo com HIV/AIDS quanto ao número de filhos ajustado por idade (Tabela 3); $30,2 \%$ de mulheres vivendo com HIV/AIDS relataram ter três ou mais filhos na época da infecção e 27,6\% das mulheres não vivendo com HIV/ AIDS tinham este mesmo número de filhos.
Quanto ao uso de preservativo, 32\% das mulheres não vivendo com HIV/AIDS relataram que nunca usavam preservativos durante as relações sexuais e $46,1 \%$ o usavam às vezes. Tais porcentagens foram significativamente maiores para as mulheres vivendo com HIV/AIDS ao se referirem à época da infecção, 62,2\% nunca usavam preservativos e $32,3 \%$ usavam às vezes $(p<0,0001)$. Mesmo não apresentado em tabela, é importante ressaltar que das mulheres vivendo com HIV/ AIDS que referiram nunca ter usado preservativo até o momento da infecção, $68,4 \%$ tinham até a 4 a série do Ensino Fundamental contra $48,4 \%$ entre mulheres não vivendo com HIV/AIDS com o mesmo grau de escolaridade ( $p<0,0001)$.

Outro dado relevante é que das mulheres vivendo com HIV/AIDS que tinham parceiro fixo com sorologia positiva $(n=509), 38,5 \%$ relataram fazer uso de preservativos de forma inconsistente (definida como nunca usar o preservativo durante as relações sexuais ou usar às vezes); esta proporção foi um pouco maior $(43,4 \%)$ para as mulheres com parceiro fixo com sorologia desconhecida $(\mathrm{n}=193)$ e significativamente menor (20\%) quando o parceiro fixo tinha sorologia negativa $(\mathrm{n}=396)$.

As mulheres vivendo com HIV/AIDS relataram uma proporção significativamente maior na prática de uso de drogas injetáveis ou não, bem como de sexo em troca de dinheiro e/ou drogas 
quando comparadas com mulheres não vivendo com HIV/AIDS conforme mostra a Tabela 3.

O histórico de DST entre mulheres vivendo com HIV/AIDS na época da infecção foi significantemente maior do que o histórico de DST alguma vez na vida entre mulheres não vivendo com HIV/AIDS (21\% contra $16,7 \%$, respectivamente; $\mathrm{p}<0,05)$ (Tabela 3).

O histórico de violência sexual alguma vez na vida também foi significativamente maior para mulheres vivendo com HIV/AIDS do que para mulheres não vivendo com HIV/AIDS (22,1\% contra $13,2 \%$, respectivamente; $\mathrm{p}<0,05$ ).

\section{Discussão}

Existem poucos estudos que analisam comparativamente o perfil de mulheres vivendo com HIV/AIDS com o de mulheres da população em geral 11,12,13. No Brasil, somente um estudo, restrito a uma cidade do Sul do país, foi identificado 14. Além disso, nenhum estudo foi encontrado na literatura pesquisada que tenha considerado na análise o contexto em que ocorreu a infecção pelo HIV. A tentativa de caracterização das situações vividas pelas mulheres vivendo com HIV/AIDS na época em que elas se infectaram é de fundamental importância, considerando-se que estes são os cenários nos quais poderiam ser identificados os fatores facilitadores da infecção pelo HIV.

A maior mediana de idade das mulheres vivendo com HIV/AIDS, assim como as idades medianas na época da infecção e na época do diagnóstico, é compatível com as faixas etárias de maior prevalência da infecção entre as mulheres notificadas com AIDS no Brasil, como também são compatíveis as proporções de raça/cor das mulheres pesquisadas com os das mulheres notificadas 1. Essas proporções podem variar nas diversas regiões do país.

Em estudo de caso-controle realizado em Porto Alegre (Rio Grande do Sul) entre 1999 e 2000, com uma amostra de mulheres vivendo com HIV/AIDS (caso) e uma amostra probabilística de base populacional de mulheres com idade entre 15 e 49 anos (controle), tanto a infecção assintomática pelo HIV como a AIDS se mostraram associadas à cor/raça negra e às mulheres com menor renda. Com relação à idade, a AIDS se mostrou associada às faixas etárias maiores do que 25 anos, enquanto a infecção assintomática pelo HIV a todas as faixas etárias 14 .

A maior proporção de mulheres vivendo com HIV/AIDS que não trabalhavam no momento da entrevista, encontrada em nosso estudo, pode estar relacionada tanto a um estado de saúde mais comprometido das primeiras, quanto às dificuldades que as pessoas vivendo com HIV/ AIDS enfrentam para ingressar e permanecer no mercado de trabalho, após seu diagnóstico. Padrão também identificado no estudo de Silveira et al. 14 .

Considerando as formas de conhecimento do diagnóstico da infecção pelo HIV citadas pelas mulheres vivendo com HIV/AIDS, verifica-se que mais da metade das mulheres conheceu seu status sorológico em decorrência do diagnóstico de AIDS no companheiro, nela própria, ou em um filho, $30 \%$ realizaram o teste por demanda do profissional de saúde e apenas $12,7 \%$ fizeram o teste por iniciativa própria. Esses dados denotam a baixa percepção de risco dessas mulheres com relação à infecção pelo HIV, observada também em outros estudos 15,16. Isto tem implicações importantes tanto do ponto de vista clínico, pois a baixa percepção de risco leva a diagnósticos tardios, quanto do ponto de vista epidemiológico, porque a transmissão sexual do HIV para uma segunda pessoa ocorre com maior freqüência antes de a primeira ter conhecimento de estar infectada pelo HIV 17.

A alta proporção, encontrada neste estudo, de mulheres infectadas pelos seus parceiros fixos e de uso inconsistente de preservativos pelas mulheres vivendo com HIV/AIDS sugerem não apenas uma possível baixa percepção de risco, como também impossibilidade de negociar de modo efetivo o uso do preservativo ou os termos da relação afetiva, ou ainda de sair dela 18 .

Estudo realizado com mulheres casadas na Região Metropolitana de Recife, por exemplo, revela que as mulheres identificam o casamento como uma proteção para a infecção pelo HIV e algumas não conhecem a etiologia da AIDS e os modos de transmissão do HIV, apresentando ainda uma visão da AIDS como doença dos outros 19.

A comparação dos dados das mulheres vivendo com HIV/AIDS e das mulheres não vivendo com HIV/AIDS também demonstrou alguns aspectos interessantes quanto ao comportamento sexual. As mulheres vivendo com HIV/AIDS não apresentaram uma diferença estatisticamente significante quanto ao número mediano de parceiros sexuais na vida, em comparação com as mulheres não vivendo com HIV/AIDS; e embora uma proporção um pouco maior de mulheres vivendo com HIV/AIDS tenha referido quatro ou mais parceiros sexuais até o momento da infecção, a diferença desapareceu quando foram excluídas da análise as mulheres que relataram ter feito sexo em troca de dinheiro e/drogas.

É oportuno destacar que inquéritos populacionais sobre comportamento e atitudes frente 
ao crescimento da AIDS no Brasil realizados até 2005 mostraram aumento do uso de preservativo, especialmente nas parcerias eventuais, mas pouca alteração de outras dimensões do comportamento sexual da maioria das pessoas ${ }^{20}$. Ao mesmo tempo, e contraditoriamente, estudo de opinião encontrou que a redução do número de parceiros, a manutenção de parcerias sexuais exclusivas, ou ainda exigência de fidelidade foram as estratégias de prevenção mais citadas $(40 \%$ dos homens e mulheres) 21 .

A orientação de diminuir o número de parceiros ou permanecer em relações monogâmicas como forma de prevenção das DST/AIDS não tem se mostrado eficaz, haja vista a grande parcela das mulheres infectadas pelo HIV por via heterossexual que referiu parceria sexual única no momento da infecção neste estudo e do diagnóstico em estudo anterior 22.

Algumas diferenças entre as mulheres vivendo com HIV/AIDS e as mulheres não vivendo com HIV/AIDS mostraram significância estatística e devem ser ressaltadas: uso de drogas; início da vida sexual mais precoce; maior proporção de mulheres que não fazia uso de preservativos, que relataram histórico de DST e de violência sexual entre as mulheres vivendo com HIV/AIDS, são fatores de extrema importância e que potencializam o risco de infecção pelo HIV.

A presença de DST é um fator importantíssimo para o aumento da possibilidade de transmissão do HIV. Dois países africanos conseguiram reduzir a incidência desta infecção com programas de tratamento em massa das DST. Em Masaka, Uganda, a prevalência de HIV em mulheres de 20 a 24 anos decresceu de 20,9\% em 1989/1990 a 13,8\% em 1996/1997. Em Zâmbia, a soroprevalência passou de $27 \%$ em 1993 a 17\% em 199823 entre mulheres assistidas em clínicas de pré-natal, de 15 a 19 anos. Cabe ressaltar que mesmo em países industrializados não se obteve uma redução desta magnitude por meio de programas de prevenção.

A pesquisa de opinião ${ }^{21}$, referida anteriormente, mostrou que a dificuldade de uso de preservativos não está ligada ao desconhecimento das formas de transmissão do HIV ou à falta de informação sobre a eficácia do preservativo. A maioria dos entrevistados diz estar ciente do risco de se infectar, mesmo com parceiros fixos, e concorda com a idéia de que mesmo as mulheres casadas e aquelas que têm namorado fixo correm um alto risco de pegar AIDS porque seus parceiros mantêm outras relações que as mulheres desconhecem 21. Concordam, igualmente, com a opinião de que as mulheres não conseguem convencer os parceiros a usar sempre camisinha. A reconhecida resistência dos homens ao uso do preservativo deixa as mulheres mais vulneráveis neste contexto.

Não se pode discutir a AIDS e seu enfrentamento sem compreender as relações sociais de gênero e suas implicações nas interações afetivas e sexuais entre homens e mulheres. A adoção de medidas preventivas, como é o caso do preservativo, esbarra em temas sobre os quais é difícil dialogar e exige a superação de barreiras sociais, culturais e emocionais. Se ultrapassar esses obstáculos é um processo árduo, ao mesmo tempo é necessário para que a ameaça real à vida de milhares de homens, mulheres e crianças em todo o mundo seja superada 9 .

A estruturação das relações de gênero pela dominação e desqualificação das mulheres propicia e naturaliza as desigualdades de poder nas relações afetivas, conjugais e/ou intimas, dificultando a abordagem de questões relativas à sexualidade, fidelidade e proteção. De igual modo naturalizam a violência, sobretudo a sexual, contra as mulheres, cujos corpos passam a ser tomados como objetos do desejo masculino 24 .

\section{Conclusões}

Ao contrário do imaginário social, as mulheres vivendo com HIV/AIDS não apresentam um número de parceiros significativamente diferente com relação às mulheres não vivendo com HIV/ AIDS. O uso de drogas, o início da vida sexual mais precoce, a baixa aderência ao uso de preservativos, a maior proporção de histórico de DST e de violência sexual entre as mulheres vivendo com HIV/AIDS, são fatores que apresentaram diferenças significantes do ponto de vista estatístico entre os dois grupos pesquisados e apontam algumas questões que a sociedade e os serviços de saúde necessitam enfrentar para diminuir a vulnerabilidade das mulheres à infecção pelo HIV. A alta proporção de mulheres infectadas por seus parceiros fixos aponta a necessidade urgente de implementar estratégias de prevenção voltadas para esta parcela da população.

Diante das questões salientadas é indispensável buscar formas de enfrentamento e prevenção da infecção pelo HIV que atendam as demandas das mulheres em suas necessidades físicas, psicológicas e culturais, considerando-se o envolvimento dos homens como parte fundamental desse processo 25 . Só será possível obter qualquer avanço nas discussões e propostas de prevenção se as expectativas de mulheres e homens, no que diz respeito à sexualidade e reprodução, forem consideradas, e se houver coragem por parte dos gestores e formuladores de políticas públicas de 
flexibilizar a discussão sobre o enfrentamento da epidemia do HIV entre as mulheres.

Ainda que o método sabidamente mais seguro para evitar a transmissão sexual do HIV seja o uso de preservativos em todas as relações sexuais, o discurso da prevenção não pode ser cristalizado, uma vez que mesmo de posse do conhecimento necessário para entender esta proposta, a maioria das pessoas não consegue praticá-la. Tanto porque o uso regular de preservativos é dificultado não só pelas relações de gênero, mas também, e talvez fundamentalmente, pelas relações de afeto e desejo, quanto porque ele inviabiliza a possibilidade de reprodução humana.

Assim, é urgente enfrentar a discussão relativa à adoção de propostas alternativas que possam diminuir a possibilidade da transmissão sexual do HIV, tais como: discutir a possibilidade de acordos entre casais estáveis, nos quais ambos fazem o teste anti-HIV, com alguma periodicidade e, se negativos, têm relações sexuais sem preservativos, comprometendo-se a usá-los nas relações com parceiros sexuais eventuais 26; considerar a função de dupla proteção do preservativo masculino, estimulando seu uso enquanto contraceptivo, tornando a prevenção às DST/ HIV mais aceitável e garantindo o acesso efetivo e ágil à contracepção de emergência nos casos de falha ou de não-uso deste insumo; discutir a possibilidade do uso do preservativo feminino e de relações sem penetração como medidas de prevenção da infecção pelo HIV. Além disso, informar de maneira clara que a presença de qualquer lesão genital aumenta o risco de aquisição do HIV e investir fortemente em capacitação dos profissionais e serviços tanto especializados em DST/AIDS, quanto de atendimento à saúde da mulher para tratamento sindrômico das DST e demais lesões genitais.

Explicitar todas as possibilidades de minimização de risco conhecidas constitui sem dúvida caminho a trilhar de forma a permitir que as pessoas façam escolhas mais condizentes com a realidade de suas relações sexuais e afetivas, tal como proposto pelo modelo hierárquico de prevenção 27 .

Os gestores e profissionais da área de saúde dos programas de DST/AIDS e da saúde da mulher devem entender a violência contra a mulher como um problema de saúde pública, que pode ter severas conseqüências na saúde física e psíquica das pessoas afetadas, entre as quais as DST, a infecção pelo HIV e a AIDS. Por outro lado, é necessário reconhecer igualmente que os diversos fatores implicados na violência sexual e doméstica exigem ações inter-setoriais para o seu enfrentamento que vão além das atividades do setor saúde e demandam parcerias efetivas, principalmente com a justiça e a segurança pública 28.

\section{Resumo}

Este artigo tem como objetivo identificar os contextos de vulnerabilidade para o HIV entre mulheres brasileiras. Entre novembro de 2003 a dezembro de 2004 foi realizado um estudo de corte transversal em 13 municípios distribuídos nas cinco regiões do país, incluindo, respectivamente, 1.777 mulheres com diagnóstico positivo para HIV e 2.045 mulheres usuárias de serviços públicos de atenção à saúde da mulher sem diagnóstico conhecido de soropositividade para o HIV. A comparação entre os dois grupos mostrou que as mulheres com diagnóstico de HIVIAIDS não apresentaram um número de parceiros significativamente diferente com relação às mulheres sem diagnóstico de HIVIAIDS. No entanto, as mulheres vivendo com HIVIAIDS apresentaram início da vida sexual mais precoce, menor aderência ao uso de preservativos, e uma maior proporção dessas mulheres relatou uso de drogas, ocorrência de DST e de violência sexual na vida. Tais resultados sugerem a importância de pensar em estratégias de prevenção voltadas para o fortalecimento das mulheres e não apenas focadas em seus comportamentos individuais.

Vulnerabilidade; HIV; Síndrome de Imunodeficiência Adquirida; Mulheres 


\section{Colaboradores}

N. J. S. Santos, R. M. Barbosa e A. A. Pinho trabalharam na análise e interpretação dos dados, e na redação final do artigo. E. V. Filipe, W. V. Vilella e N. J. S. Santos colaboraram na concepção e delineamento do estudo e na redação final do artigo. T. Aidar revisou a análise e trabalhou na redação final do artigo.

\section{Agradecimentos}

O estudo teve financiamento do Programa Nacional de DST/AIDS e do Centro de Referência e Treinamento DST/AIDS da Secretaria Estadual de Saúde de São Paulo; a análise dos dados foi realizada por meio de financiamento do Conselho Nacional de Desenvolvimento Científico e Tecnológico (CNPq), pelo Edital 22/2007 - Saúde da Mulher - Linha de Apoio 1.2.1.

\section{Referências}

1. Ministério da Saúde. Boletim Epidemiológico de DST e AIDS 2007/2008; ano V, no. 1.

2. Santos NJS, Tayra A, Silva SR, Buchalla CM, Laurenti R. A AIDS no Estado de São Paulo. As mudanças no perfil da epidemia e perspectivas da vigilância epidemiológica. Rev Bras Epidemiol 2002; 5:286-310.

3. Plummer FA, Moses S, Ndinya-Achola JO. Factors affecting female-to-male transmission of HIV-1: implications of transmission dynamics for prevention. In: Chen LC, Amor JS, Segal SJ, editors. AIDS and women's reproductive health. New York: Plenum Press; 1991. p. 3-45.

4. Downs AM, De Vicenzi I. Probability of heterosexual transmission of HIV: relationship to the number of unprotected sexual contacts. European Study Group in Heterosexual Transmission of HIV. J Acquir Immune Defic Syndr Hum Retrovirol 1996; 11:388-95.
5. Ronald AR. Slowing heterosexual HIV transmission. Infect Dis Clin North Am 1995; 9:287-96.

6. Giffin K. Beyond empowerment: heterosexualities and the prevention of AIDS. Soc Sci Med 1998; 46:151-6.

7. Vermelho L. Women with AIDS: transmission and prevention strategies. In: Proceedings of the 12th World AIDS Conference. Geneva: United Nations Joint Programme on HIV/AIDS; 1998. p. 440-1.

8. Villela W. O impacto social e econômico do HIV/ AIDS no Brasil. In: Pitanguy J, Mota A, organizadoras. Os novos desafios da responsabilidade política. Rio de Janeiro: Cidadania, Estudo, Pesquisa, Informação e Ação; 2005. p. 79-95. (Cadernos Fórum Civil, ano 7, no. 6).

9. Barbosa RHS. AIDS e saúde reprodutiva: novos desafios. In: Giffin K, Costa SH, organizadores. Questões de saúde reprodutiva. Rio de Janeiro: Editora Fiocruz; 1999. p. 281-96. 
10. Bajos N, Lamarche-Vadel A, Gilbert F, Ferrand M, Moreau C, Bouyer J, et al. Contraception at the time of abortion: high-risk time or high-risk women? Hum Reprod 2006; 21:2862-7.

11. Tran TT, Mulhall BP, Macaskill P, Nguyen TQ. Risk factors for HIV infection in a gynaeco-obstetric population in Vietnam: a case control study. Sex Health 2005; 2:71-5.

12. Wyatt GE, Myers HF, Williams JK, Kitchen CR, Loeb T, Carmona JV, et al. Does a history of trauma contribute to HIV risk for women of colour? Implications for prevention and policy. Am J Public Health 2002; 92:660-5.

13. Bates I, Fenton C, Gruber J, Lalloo D, Lara AM, Squire SB, et al. Vulnerability to malaria, tuberculosis and HIV/AIDS infection and disease. Part 1: determinants operating at individual and household level. Lancet Infect Dis 2004; 4: 267-77.

14. Silveira MF, Santos IS, Victora CG. Poverty, skin colour and HIV infection: a case-control study from southern Brazil. AIDS Care 2008, 20:267-72.

15. Braga PE, Cardoso MRA, Segurado AC. Diferenças de gênero ao acolhimento de pessoas vivendo com HIV em serviço universitário de referência de São Paulo, Brasil. Cad Saúde Pública 2007; 23:2653-62.

16. Filipe EMV, Bugamelli L, Leme B, Santos NJS, Garcia S, Paiva V, et al. Risk perception and counselling among HIV-positive women in São Paulo, Brazil. Int J STD AIDS 2000; 11:112-4.

17. Filipe EMV, Batistella E, Pine A, Santos NJS, Paiva V, Segurado A, et al. Sexual orientation, use of drugs and risk perception among HIV-positive men in São Paulo, Brasil. Int J STD AIDS 2005; 16:56-60.

18. Barbosa RM. Negociação sexual e sexo negociado, sexualidade e gênero em tempos de AIDS [Tese de Doutorado]. Rio de Janeiro: Universidade Federal do Rio de Janeiro; 1997.
19. Nascimento AMG, Barbosa CS, Medrado B. Mulheres em Camaragibe: representação social sobre a vulnerabilidade feminina em tempos de AIDS. Rev Bras Saúde Matern Infant 2005; 5:77-86.

20. Barbosa RM, Koyama MAH. Comportamento e práticas sexuais de homens e mulheres, Brasil 1998 e 2005. Rev Saúde Pública 2008; 42:21-33.

21. Instituto Patrícia Galvão. Atitudes frente ao crescimento da AIDS no Brasil. São Paulo: Instituto Patrícia Galvão; 2003.

22. Santos NJS, Buchalla CM, Filipe EMV, Bugamelli L, Garcia S, Paiva V. Mulheres HIV positivas, reprodução e sexualidade. Rev Saúde Pública 2002; 36: 12-23.

23. Centers for Disease Control and Prevention. The global HIV and AIDS epidemic, 2001. MMWR Morb Mortal Wkly Rep 2001; 50:434-9.

24. Villela W. Mulher, violência e AIDS: explorando interfaces. In: Nilo A, organizador. Mulher, violência e AIDS. Recife: GESTOS - Soropositividade, Comunicação \& Gênero; 2008. p. 107-26.

25. Arilha M. Homens, saúde reprodutiva e gênero: o desafio da inclusão. In: Giffin K, Costa SH, organizadores. Questões de saúde reprodutiva. Rio de Janeiro: Editora Fiocruz; 1999. p. 455-67.

26. Kippax S, Noble J, Prestage G, Crawford JM, Campbell D, Baxter D, et al. Sexual negotiation in the AIDS era: negotiated safety revisited. AIDS 1997; 11:191-7.

27. Barbosa RM. HIV/AIDS, transmissão heterossexual e métodos de prevenção controlados pelas mulheres. Rio de Janeiro: Associação Brasileira Interdisciplinar de AIDS; 2001. (Coleção ABIA. Saúde Sexual e Reprodutiva, 2).

28. Coordenação Nacional de DST/AIDS, Secretaria de Programas de Saúde, Ministério da Saúde. Políticas e diretrizes de prevenção das DST/AIDS entre mulheres. Brasília: Ministério da Saúde; 2003.

\footnotetext{
Recebido em 07/Jan/2009

Versão final reapresentada em 24/Jun/2009

Aprovado em 26/Jun/2009
} 